OPEN ACCESS

Edited by:

Ruixin Zhu,

Tongji University, China

Reviewed by:

Mingfeng Xia,

Fudan University, China

Jing Yuan,

Capital Institute of Pediatrics, China

*Correspondence:

Jing-Pu Zhang

zhangjingpu@imb.pumc.edu.cn

Chang-Qin Hu

hucq@nifdc.org.cn

${ }^{t}$ These authors have contributed equally to this work

Specialty section:

This article was submitted to

Gastrointestinal and

Hepatic Pharmacology,

a section of the journal

Frontiers in Pharmacology

Received: 20 September 2019 Accepted: 20 November 2019

Published: 08 January 2020

Citation:

Chen $B$, Zheng $Y-M$, Zhang $M-Q$,

Han Y, Zhang J-P and Hu C-Q (2020) Microarray Expression Profiling and Raman Spectroscopy Reveal Anti-

Fatty Liver Action of Berberine in a Diet-Induced Lanval Zebrafish Model.

Front. Pharmacol. 10:1504.

doi: 10.3389/fphar.2019.01504

\section{Microarray Expression Profiling and Raman Spectroscopy Reveal Anti- Fatty Liver Action of Berberine in a Diet-Induced Larval Zebrafish Model}

\author{
Bo Chen ${ }^{1,2 \dagger}$, Yang-Min Zheng ${ }^{1,2,3+}$, Miao-Qing Zhang ${ }^{1,2,4,5}$, Ying Han ${ }^{6}$, Jing-Pu Zhang ${ }^{1,2 *}$ \\ and Chang-Qin $\mathrm{Hu}^{6 *}$ \\ 1 Key Laboratory of Biotechnology of Antibiotics, The National Health Commission (NHC), Institute of Medicinal \\ Biotechnology, Chinese Academy of Medical Sciences \& Peking Union Medical College, Beijing, China, ${ }^{2}$ Beijing Key \\ Laboratory of Antimicrobial Agents, Institute of Medicinal Biotechnology, Chinese Academy of Medical Sciences \& Peking \\ Union Medical College, Beijing, China, ${ }^{3}$ Institute of Cerebrovascular Disease Research, Xuanwu Hospital of Capital Medical \\ University, Beijing, China, ${ }^{4}$ Postdoctoral Scientific Research Workstation, China Resources Sanjiu Medical \& Pharmaceutical \\ Co., Ltd., Shenzhen, China, ${ }^{5}$ Postdoctoral Mobile Research Station, Institute of Process Engineering, Chinese Academy of \\ Sciences, Beijing, China, ${ }^{6}$ National Institutes for Food and Drug Control, Graduate School of Peking Union Medical College, \\ Beijing, China
}

Background: The prevalence of non-alcohol fatty liver disease (NAFLD) is increasing in children and adolescents who are mostly resulted from overfeeding. Previous studies demonstrate that berberine (BBR), a compound derived from plant, has beneficial effects on NAFLD in adults but poorly understood in the pediatric population. This study employed a larval zebrafish model to mimic the therapeutic effects of BBR in the pediatric population and the mechanisms underlying its hepatoprotection.

Methods: High-cholesterol diet (HCD)-fed zebrafish exposed to BBR at doses of 0, 1, 5, and $25 \mu \mathrm{M}$. After the larvae were treated with BBR for 10 days, its effect on hepatic steatosis was evaluated. We introduced Raman imaging and three-dimensional (3D) molecular imaging to detect changes in the biochemical composition and reactive oxygen species (ROS) levels of zebrafish liver. Gene expression microarray was performed to identify differentially expressed genes (DEGs) followed by gene ontology (GO), Kyoto Encyclopedia of Genes and Genomes (KEGG) pathway, and functional category analysis.

Results: BBR (5 and $25 \mu \mathrm{M}$ ) administration prevented HCD-induced liver lipid accumulation in larval zebrafish. The result was further confirmed by the pathological observation. Raman mapping indicated that the biochemical composition in the liver of BBR-treated group shifted to the control. The quantitative analysis of 3D imaging showed that the ROS level was significantly decreased in the liver of BBR-treated larvae. In the livers of the BBR group, we found 468 DEGs, including 172 genes with upregulated expression and 296 genes with downregulated expression. Besides, GO enrichment, KEGG pathway, and functional category analysis showed that various processes related to glucolipid metabolism, immune response, DNA damage and repair, and iron were 
significantly enriched with DEGs. The expression levels of the crucial genes from the functional analysis were also confirmed by quantitative PCR (qPCR).

Conclusion: BBR can significantly improve hepatic steatosis in HCD-fed zebrafish larvae. Its mechanisms might be associated with the regulation of lipid metabolism, oxidative stress, and iron homeostasis. Raman imaging in larval zebrafish might become a useful tool for drug evaluation. Mainly, the gene expression profiles provide molecular information for BBR on the prevention and treatment of pediatric NAFLD.

Keywords: berberine, non-alcohol fatty liver disease, Raman mapping, larval zebrafish, transcriptomics

\section{INTRODUCTION}

Non-alcohol fatty liver disease (NAFLD) is characterized by excessive fat accumulation in liver cells, which is cumulatively prevalent due to the worldwide obesity epidemic (Wruck et al., 2017). NAFLD is a risk factor of metabolic diseases, such as type 2 diabetes, obesity, and dyslipidemia (Mahfood Haddad et al., 2017). Indeed, NAFLD can progress to its more severe form nonalcoholic steatohepatitis (NASH) (Day and James, 1998), fibrosis, and may eventually develop into hepatocellular carcinoma (Day, 2006). In general, NAFLD was considered a disease of adults. However, the prevalence of NAFLD is rapidly increasing in children and adolescents, especially in obese children (Hatton et al., 2018). The treatment approach is limited because of unclear pathogenesis of NAFLD and lack of therapeutic agents. Therefore, it is reasonable to develop suitable treatment strategies for children suffering from this disease (BetancourtGarcia et al., 2017; Assuncao et al., 2017).

Berberine (BBR) is an isoquinoline alkaloid, source of berberis, and has been widely used in traditional Chinese medicine for hundreds of years. Modern pharmacology demonstrates that BBR has antibacterial, anti-diabetic, antihyperlipidemic, and multiple cardioprotective effects (Imenshahidi and Hosseinzadeh, 2019). Many studies showed that BBR could regulate metabolism disorder, increase insulin sensitivity, and improve glycometabolism, which may indicate it has potential in the treatment of NAFLD (Zhu et al., 2016). However, most of the animal experiments and clinical trials of BBR were executed in adult animals and the adult population. The histological features of NAFLD/NASH in children and adults are quite different (Nobili et al., 2006). Childhood NASH exhibits a much more common predominance of portal inflammation than in adult NASH, yet the differences of the mechanisms between adults and children in the pathogenesis of NAFLD are poorly understood (Nobili et al., 2016; Hatton et al., 2018). On the other hand, there is a difference in sensitivity to medications between children and adults (Assuncao et al., 2017). So, it is necessary to explore the effects and mechanisms of BBR on NAFLD in children and young animal models.

Zebrafish are a small vertebrate organism that has been applied for in vivo models in various fields including genetics, developmental biology, toxicology, and preclinical medicine experiments (Barros et al., 2008; Albadri et al., 2017; Chen et al., 2017). Zebrafish are easy to breed and mature fast as well as cost little in daily maintenance. Recent studies demonstrated that zebrafish has a similar process with humans in lipid metabolism (Holtta-Vuori et al., 2010). Moreover, because the zebrafish larvae have optical transparency, the livers can be easily observed in real time (Schlegel, 2012). Recently, zebrafish have become increasingly popular in research of pediatric diseases, such as acute leukemia models and infectious diseases (Veldman and Lin, 2008; Lohi et al., 2013). Several zebrafish models in both adults and larvae have been well established (Gu et al., 2014; Sapp et al., 2014; Dai et al., 2015; Chen et al., 2018) to study NAFLD. That makes it possible to evaluate the drugs in the larvae. Zebrafish have therefore been used as alternatives to rodents for animal models in the present study.

Herein, we constructed diet-induced steatosis in zebrafish larvae that could mimic human children's disease. In particular, the role of BBR on NAFLD in the larval zebrafish model remains to be determined. This study aimed to evaluate the effects and mechanisms of BBR on hepatic steatosis and provide data support for BBR in the treatment of pediatric disease. To get further insights into the molecular mechanism for the anti-fatty liver action of BBR, we performed microarray analysis to define the differences in the transcriptome of liver from diet-induced zebrafish larvae treated with or without BBR and validated the expression levels of the crucially differentially expressed genes (DEGs) that were identified by functional category analysis. Furthermore, we introduced Raman confocal microspectroscopy and three-dimensional (3D) molecular imaging to analyze the changes of biochemistry component and reactive oxygen species (ROS) levels in the larval liver, respectively.

\section{METHODS AND MATERIALS}

\section{Ethical Approval}

This study was reviewed and approved by the Laboratory Animal Management and Animal Welfare Committee in the Institute of Medicinal Biotechnology, Chinese Academy of Medical Sciences, and we always made the most effort to minimize animals' suffering.

\section{Zebrafish Care and Treatment}

$\mathrm{AB}$ strain zebrafish (Danio rerio) and Tg (fabp10a: dsRed) zebrafish were kept under standard laboratory conditions with a 14-h light/10-h dark cycle at a temperature of $28.5 \pm 1^{\circ} \mathrm{C}$ 
(Hu et al., 2011). Embryos and larvae were maintained in embryo medium to 5 days post-fertilization (dpf) without feeding. Zebrafish larvae were fed with control diet (AP100, $30 \mathrm{mg} / 100$ fish/day) or a high-cholesterol diet (HCD, $180 \mathrm{mg}$ AP100 plus $4 \%$ cholesterol/100 fish/day) for 10 days. For hepatic steatosis analysis, larvae in the HCD group were exposed to BBR at doses of $0,1,5$, and $25 \mu \mathrm{M}$ for a 10 -day treatment period until $15 \mathrm{dpf}$. The doses selected in this study were referred to the ones used in previous studies (Zheng et al., 2018). According to the results of hepatic steatosis analysis, $5 \mu \mathrm{M}$ of $\mathrm{BBR}$ was applied to the subsequent experiments, including histologic analysis, Raman measurement, ROS detection, microarray analysis, and quantitative PCR (qPCR).

\section{Whole-Mount ORO Staining}

Zebrafish larvae were fixed in $4 \%$ paraformaldehyde (PFA) overnight at $4^{\circ} \mathrm{C}$ and washed twice with phosphate-buffered saline (PBS). Oil Red O (ORO) staining was performed as described before (Sapp et al., 2014). Lipid droplets in liver tissue were observed and imaged on a bright-field dissecting microscope (Olympus szx10, Tokyo, Japan). Larvae were defined as positive for steatosis according to the previous report (Sapp et al., 2014).

\section{Histologic Analysis}

Larvae were fixed with $4 \%$ PFA overnight, coated in paraffin, and cut into $4-\mu \mathrm{m}$ slices with paraffin microtome. The sections were stained with hematoxylin and eosin (H\&E). For ORO staining of cryosections, larvae were embedded in OCT (Sakura Japan Co., Ltd., Tokyo, Japan) and stored at $-80^{\circ} \mathrm{C}$ until sectioning. Serial sections $(8 \mu \mathrm{m})$ were cut. Each section was stained with $0.5 \%$ ORO. Slides were observed using an Olympus BX53 microscope (Olympus, Tokyo, Japan). The positive area of ORO was calculated with ImageJ software.

\section{Electron Microscopy}

After the experiment, zebrafish larvae were collected and fixed in buffered $2 \%$ glutaraldehyde. Briefly, the fixed larvae were mounted and sectioned. Then the slides were imaged by a transmission electron microscope (Tecnai Spirit, FEI, USA) as in previous studies (EauClaire et al., 2012).

\section{Biological Analysis}

Levels of total cholesterols (TCs) and triglycerides (TGs) of the dissected liver of zebrafish larvae were determined by Total Cholesterol Reagent Kit and Triglyceride Reagent Kit (Applygen Technologies Inc., Beijing, China) according to the manufacturer's specification, respectively. The glutathione (GSH) and malondialdehyde (MDA) levels of zebrafish tissue were determined by using the respective reagent kits according to the manufacturer's specification (Nanjing Jiancheng Biotechnology Institute, Nanjing, China).

\section{Raman Measurements of Liver Tissues}

Raman mapping was performed by using a $780-\mathrm{nm}$ wavelength excitation laser with a $\times 50-\mu \mathrm{m}$ pinhole for confocality (DXRxi Raman imaging microscope, Thermo Fisher Scientific Inc.,
Hudson, USA). The laser power and accumulation time were set at $10 \mathrm{~mW}$ and $60 \mathrm{~s}$, respectively. Raman spectra were collected over a scanning spectral range of $600-3,200 \mathrm{~cm}^{-1}$. Each liver of zebrafish was determined under the microscope at least three different regions for at least three times to acquire the average spectrum. System control and spectrum acquisition were conducted using OMNIC software (Thermo Fisher Scientific Inc., Hudson, USA). Zebrafish larvae were anesthetized with tricaine (Sigma, USA) before the Raman experiment.

\section{D Imaging to Detect ROS Level and Macrophage of the Whole Liver}

Live $\mathrm{Tg}$ (fabp10a: dsRed) zebrafish were incubated with CellROX $^{\circledR}$ Green (ThermoFisher, USA), a ROS sensor, at a final concentration of $10 \mu \mathrm{M}$ for $1 \mathrm{~h}$ in the dark, washed in PBS twice, anesthetized using tricaine (Sigma, USA), and fixed with PFA. For macrophage detection, larvae were incubated with the L-plastin antibody (Santa Cruz, USA, 1:500 dilution) at $4^{\circ} \mathrm{C}$ overnight, and then with an Alexa Fluor488-conjugated antibody (ZSGB-BIO, Beijing, China, 1:1,000 dilution) at room temperature for $2 \mathrm{~h}$. Then the fish liver was isolated and placed upon the $\times 10$ detection lens of the Deltavision widefield deconvolution imaging system (DV; GE Healthcare, USA). After image acquisition, a 3D view of the liver was generated from original data for quantitative analysis by using softWoRx (GE Healthcare, USA). All objects of interest were collected and quantified during the construction of this $3 \mathrm{D}$ model.

\section{Microarray Expression Profiling and Bioinformatic Analysis}

Total RNA was extracted using TAKARA RNAiso Plus according to the manufacturer's instructions, followed by RNA quality evaluation by Agilent Bioanalyzer 2100 (Agilent Technologies, Santa Clara, CA, USA). The Agilent zebrafish (V3) gene expression microarray $4 \times 44 \mathrm{~K}$ (Design ID 026437) was used for microarray analysis. The process of microarray scanning was carried out by CapitalBio Corporation (Beijing, China) as previously described (Han et al., 2018). To identify DEGs between the control, HCD, and BBR-treated group, the threshold of fold change $\geq 2$ was established in our analysis. Then, based on the KEGG and GO databases, biomedical pathways were classified (Kanehisa and Goto, 2000). Functional category analysis was performed by the Database for Annotation, Visualization, and Integrated Discovery (DAVID) bioinformatics program (http://david.abcc.ncifcrf.gov/) under a significance threshold of $P<0.05$. We used the Cytoscape software to map the gene-pathway interaction network (http:// www.cytoscape.org/), the shinyCircos software to map the Circos plot (Yu et al., 2018), and TBtools software to map the heatmap plot (https://www.biorxiv.org/content/10.1101/289660v1). The microarray data were deposited in Gene Expression Omnibus (GEO accession: GSE136436).

\section{Quantitative RT-PCR}

Total RNA was isolated from dissected livers of zebrafish larvae by TRIzol reagent (no.15596026, Thermo Fisher, USA) and then converted into complementary DNA (cDNA) by reverse 
transcriptase (no. M1701, Promega, USA). The cDNAs were then used as the templates for quantitative real-time polymerase chain reaction (qPCR). The reaction of $\mathrm{qPCR}$ was performed by the real-time quantification system (ROCHE LightCycler 96, Switzerland). The relative amount of messenger RNAs (mRNAs) was calculated with $\beta$-actin mRNA as the invariant control. The relative transcript expression level was determined using the control sample as a calibrator and the ${ }^{\Delta \Delta} \mathrm{CT}$ method. The following specific primers were used for amplification in this study (Table 1).

\section{Statistical Analysis}

Data are expressed as means \pm SEM. Comparisons between multiple groups were performed using one-way ANOVA with Tukey's post hoc test. In all experiments, differences were considered statistically significant for $P<0.05$.

\section{RESULTS}

\section{The Effects of BBR on Body Weight and Body Length in HCD Diet-Fed Zebrafish Larvae}

Embryos and larvae were maintained in embryo medium to $5 \mathrm{dpf}$, and zebrafish larvae without malformation were divided into five groups randomly. For model building, 5-dpf zebrafish were treated with control (30 mg AP100/100 fish/day) or HCD (180 mg AP100 plus $4 \%$ cholesterol/100 fish/day) for 10 days. HCD diet-fed zebrafish were exposed to different doses of $\operatorname{BBR}(0,1,5$, and 25 $\mu \mathrm{M})$. After 10 days of feeding and treatment, the zebrafish were washed and collected for the following experiments. The main experimental design was graphed as Figure 1A. We firstly did general observation and measured the larvae body and length. All the BBR-treated zebrafish larvae did not exhibit obvious abnormalities and varying mortality (Figure 1E). Compared with the control group, the body length in other groups were significantly larger (Figure 1B). The body weight was significantly heavier in the HCD group than those in the control group (Figure 1C).
Meanwhile, the HCD diet-fed larvae had a higher body mass index (BMI) than the control group (Figures 1C, D). Particularly, BBR treatment significantly decreased body weight and BMI, but did not affect body length (Figures 1B-D).

\section{BBR Attenuated HCD Diet-Induced Hepatic Steatosis in Larval Zebrafish}

We then assess the effects of BBR on liver lipid accumulation in zebrafish. The whole body of zebrafish larvae was stained with ORO. HCD diet-induced significant steatosis in the liver and the incidence of steatosis in the model group were much higher than in the control group (Figure 1F). Larvae treated with BBR (5 and $25 \mu \mathrm{M}$ ) observably decreased the incidence of steatosis compared with the HCD group (Figure 1G). The results of the frozen liver section with ORO staining further confirmed the effects. The relative staining area of ORO in the model group was higher than in control. BBR treatment significantly reduced the ORO positive compared with the model group (Figures 2A, line 1, and $\mathbf{C}$ ). The paraffin section with $\mathrm{H} \& \mathrm{E}$ staining showed severe macrovesicular steatosis in the liver of HCD diet-fed zebrafish, and the liver lesion was much improved by BBR treatment (Figure 2A, line 2). Transmission electron microscopy (TEM) was employed to observe the change of hepatocyte in zebrafish larvae. Several large sizes of lipid droplets were found in the HCD group, but not in the control group. Mainly, we noticed the changes in the mitochondria morphology in the HCD group, including mitochondrial elongation, tubular, and crest broke. BBR treatment rescued HCD-induced mitochondrial damage in the hepatocyte of larval zebrafish (Figure 2A, lines 3 and 4). Then the TC, TG, and glucose levels in the isolated liver from zebrafish were carefully measured. The levels of TC, TG, and glucose were notably elevated in the liver of the model group compared with the control group, and HCD-fed zebrafish with BBR treatment significantly reduced the hepatic TC, TG, and glucose contents (Figures 2E-G). To address the inflammatory response in larvae with HCD, we used the L-plastin antibody to label macrophage (Stoletov et al., 2009). The results showed that a small amount of hepatic macrophage was present in the liver of control larvae, and

TABLE 1 | Primer for qPCR genes used in this study.

\begin{tabular}{|c|c|c|}
\hline Gene symbol & Sense primer $\left(5^{\prime}-3^{\prime}\right)$ & Antisense primer $\left(5^{\prime}-3^{\prime}\right)$ \\
\hline isca1 & GTCAGATGAGGAGGTGTTAC & CCTTGATGTTGGGATTGTTG \\
\hline fads2 & CCATCGGCACTTCCAGCATCAC & TाTCCCACCACAAAGGCGTTCAG \\
\hline mvda & CTGAACAAGTGGCATCTG & ССТТАТСАТСТСТTССАТАCG \\
\hline plcg1 & AAGCAGAGAAGTATGTGAAC & GTCGTAGTTGGAGGAGTC \\
\hline h2afx & TGTTCACCGTCTCCTTCG & AGTCTTCTTGTTGTCTCTTGC \\
\hline ube2t & GTTCTCACATCCATACAGTTATTG & AGTCTITCCATCCGTCTCC \\
\hline tk1 & CCAGACACCGTTGAGTTC & ACCAGATTCAGGATGTTACC \\
\hline$|p|$ & ATGGAATACACGGCGAGAAG & CAGTTTGCGAATGTGGAAGG \\
\hline parpbp & TTGGATGAGGAGGCATTAGC & TGGACTTGATATGTTCTTGTTGG \\
\hline rev1 & GCTCCAAGTTGACСTCTC & CGAATGTTGTAGTTCATCTCC \\
\hline msmo1 & GTTGTGCTGTAATTGAAGAC & GGTGCCAATGAAGAATCC \\
\hline
\end{tabular}




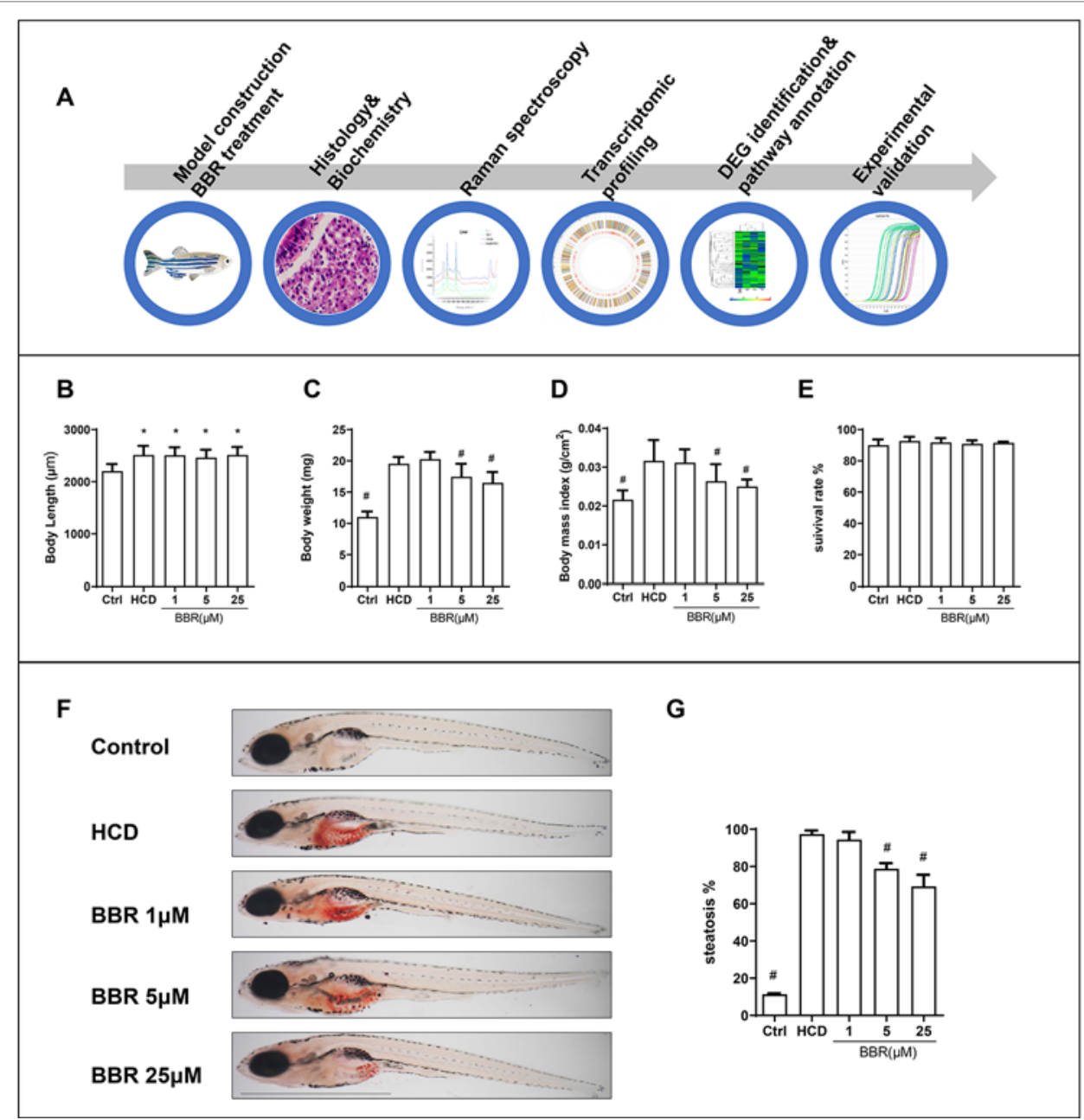

FIGURE 1 | Effects of high-cholesterol diet (HCD) and berberine (BBR) treatment on the growth of zebrafish. (A) Description of the experimental design. (B) Body length, (C) average body weight, and (D) BMl in each group were measured at 15 days post-fertilization (dpf). (E) Larvae with different treatments were scored for mortality. (F) The hepatic steatosis of zebrafish was detected by whole-body Oil Red O (ORO) staining. (G) Percent of larvae with hepatic steatosis. ${ }^{*}$ Compared with the control; " compared with the HCD group, $P<0.05$. Bar, $1 \mathrm{~mm}$.

the number of macrophages were increased in the HCD group. BBR treatment could reverse the effect of HCD on macrophage recruitment to the liver in zebrafish (Figures $2 \mathbf{B}, \mathbf{D}$ ). Furthermore, Sirius red staining was employed to assess the development of fibrosis, but no significant fibrosis was found in the three groups (Figure S1). The above results demonstrated that BBR could significantly improve diet-induced liver steatosis and exert a protective effect on hepatocyte in larval zebrafish.

\section{Raman Spectroscopy Analysis}

Raman mapping of individual liver regions of zebrafish embryos at a microresolution level was applied to provide information on their biochemical composition (Figure 3). The Raman spectra obtained from the liver of different groups exhibit differential spectral lines at around 720-790, 1,660, and 2,800-3,025 cm (Figure 3A). The Raman maps using the $\mathrm{C}-\mathrm{H}$ stretching mode in the 2,800 - to $3,025-\mathrm{cm}^{-1}$ region were associated with lipids and proteins (Majzner et al., 2014). Besides, DNA peaks can be demonstrated at 722, 752, 782, and $1,663 \mathrm{~cm}^{-1}$ (Shetty et al., 2006). Compared with the control liver, the spectra of regions at 1,663 and 2,800-3,025 $\mathrm{cm}^{-1}$ showed higher intensity in the HCD group, demonstrating an increased distribution of lipid and DNA in the liver. The results showed that BBR $(5 \mu \mathrm{M})$ had the ability to modulate liver lipid, DNA, and protein. Raman signal intensity revealed that the levels of lipid and DNA were decreased in the liver of zebrafish treated with BBR (Figure 3A). The principal component analysis (PCA) indicated the significant alterations in the biochemical composition of liver between the control and HCD groups, while the scatter plots could shift to the control group by BBR treatment, suggesting that BBR treatment has liver protection against HCD in larval zebrafish (Figure 3B). This approach further confirmed the 

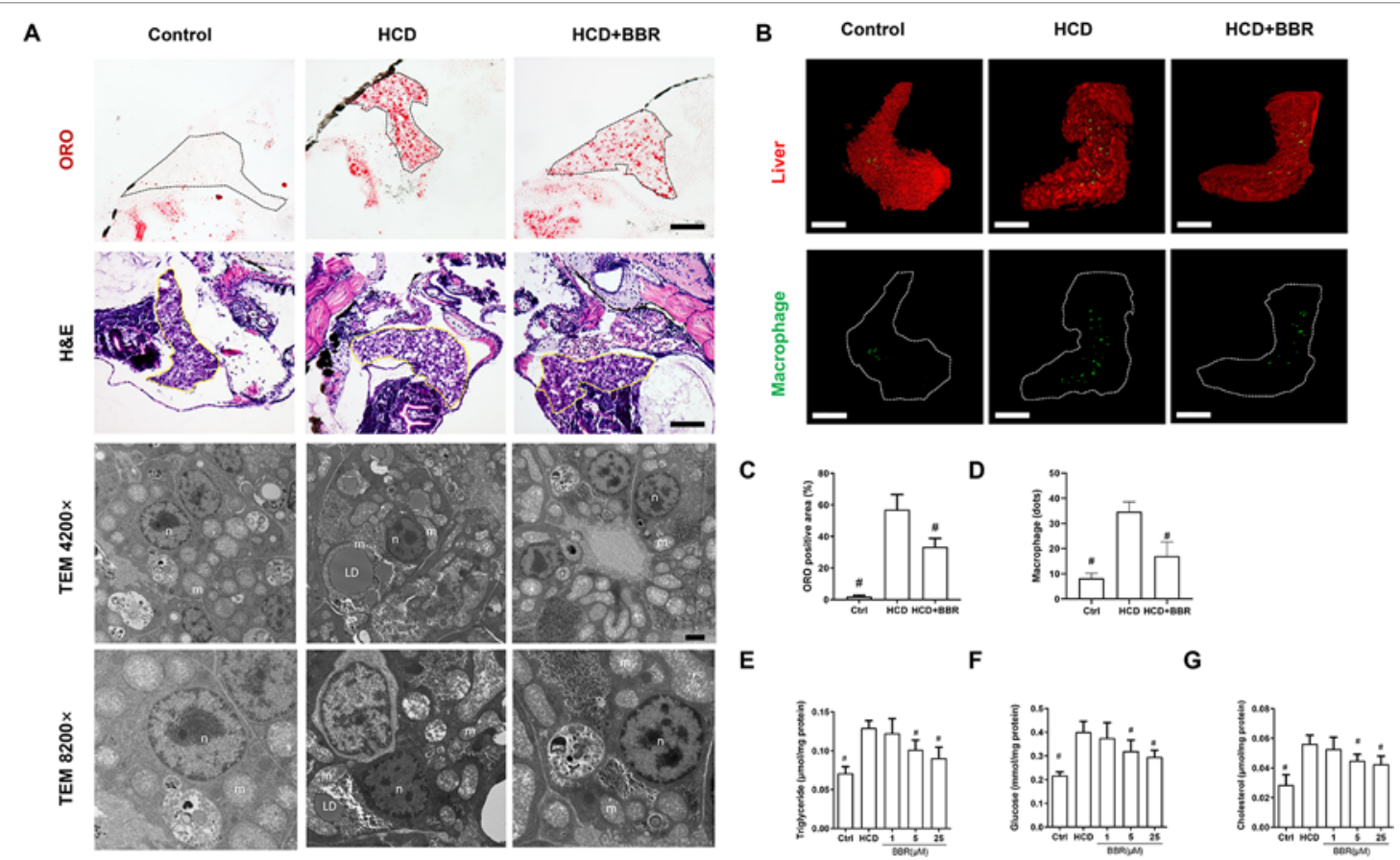

FIGURE 2 | Effects of berberine (BBR) on hepatic steatosis in high-cholesterol diet (HCD)-induced zebrafish larvae. (A) The zebrafish larvae were treated with the control diet, HCD diet, and HCD plus BBR (5 $\mu \mathrm{M})$ treatment. Hepatic steatosis was observed by whole-body Oil Red O (ORO) staining (line 1 , refer to the black dotted, bar $=100 \mu \mathrm{m}$ ), H\&E staining (line 2, refer to the yellow dotted, bar $=100 \mu \mathrm{m}$ ), and TEM (line 3 , bar $=2 \mu \mathrm{m}$; line 4, bar $=1 \mu \mathrm{m}$ ). $m$ mitochondrion, $n$ cell nucleus, LD lipid droplet. (B) L-plastin-labeled macrophages in Tg (fabp10a: dsRed) zebrafish. The statistical results of the positive area of ORO staining were shown in (C) and the quantity of macrophage in the liver of larval zebrafish in (D). Effects of BBR on the levels of triglyceride (E), glucose (F), and cholesterol (G) in livers of zebrafish larvae fed with HCD. ${ }^{\#}$ Compared with the HCD group, $P<0.05$.

histological and biochemical results and provided more detailed information on macromolecular composition.

\section{Quantification Analysis of Hepatic ROS Level Based on 3D Models of the Whole Liver}

Oxidative stress promotes the progression of NAFLD in the form of NASH (Richter and Kietzmann, 2016). The increase of cellular ROS generation results in organelle and cellular dysfunction, and apoptosis ultimately (Paradies et al., 2014; Spahis et al., 2017). Here, we introduced a method for quantitative analysis and 3D imaging, which use deconvolution algorithms, rapidly and precisely reconstructing $3 \mathrm{D}$ objects, allowing a better visual understanding of the image data and a more accurate quantitative assessment of the 3D object (Pegard and Fleischer, 2013; Chakrova et al., 2016). Compared with the control group, the HCD feeding significantly induced the hepatic ROS levels increase, which was inhibited by BBR $(5 \mu \mathrm{M})$. While BBR treatment alone did not affect the ROS generation (Figure 4A). MDA levels were significantly higher in the HCD-treated groups, whereas the GSH level was significantly lower. BBR treatment could induce the increase of GSH level and inhibit the production of MDA. No apparent changes were observed in the $5-\mu \mathrm{M}$ BBR-treated group (Figures 4C, D). These results suggested that BBR could alleviate the oxidative stress in HCD- induced zebrafish and do not affect the ROS balance in normal larval zebrafish.

\section{Global Changes in Gene Expression of Larval Zebrafish Liver}

After 10 days of treatment, the livers of zebrafish larvae (control, HCD, and BBR $5 \mu \mathrm{M}$ ) were applied to microarray analysis. The data of gene expression were obtained by using the zebrafish gene expression $4 \times 44 \mathrm{~K}$ microarray (Agilent). The array data were analyzed for data summarization, normalization, and quality control by using the GeneSpring software V13 (Agilent). To investigate the response to the HCD and BBR treatment, we performed cluster analysis. The results indicated that the BBR groups displayed closer expression patterns than the HCD groups compared to the control group. The threshold values of $\geq 2$ and -2 -fold change or less and Benjamini-Hochberg corrected $P$ value $<0.05$ were selected as the differentially expressed genes. Compared to the HCD (shortened to $M$ in Figure 5) group, 3,357 and 3,277 DEGs were identified in the control group and BBR group, respectively (Figure 5A). The DEGs with $\log 2$ (Ctrl vs. $\mathrm{M})>1$ and $\log 2(\mathrm{BBR}$ vs. $\mathrm{M})>1$ expression levels and the DEGs with $\log 2$ (Ctrl vs. M) less than -1 and $\log 2$ (BBR vs. M) less than -1 expression levels at $P<0.05$ were combined, which leads to the identification of 468 genes with significantly different expression levels in the livers of the 
A
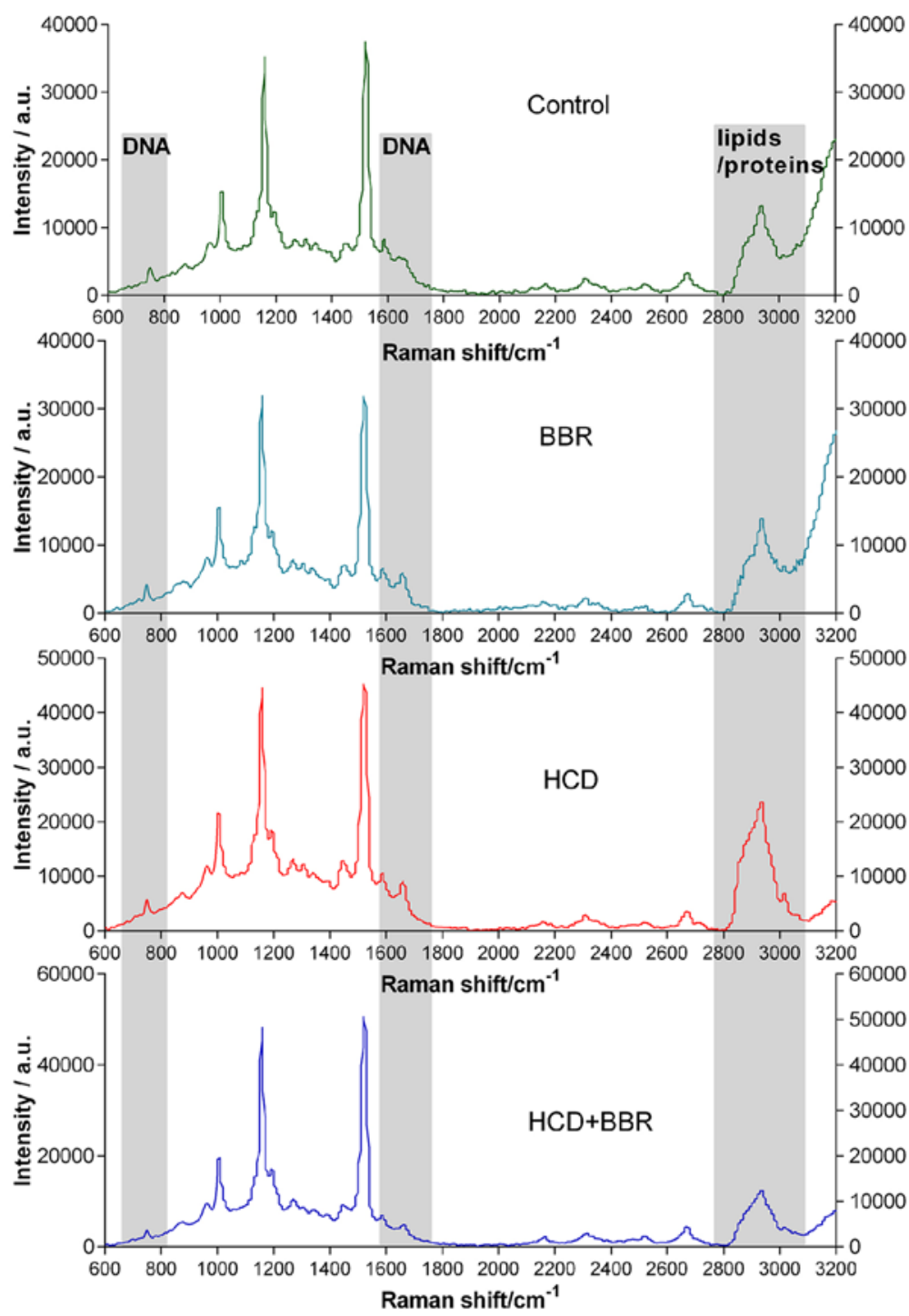

B

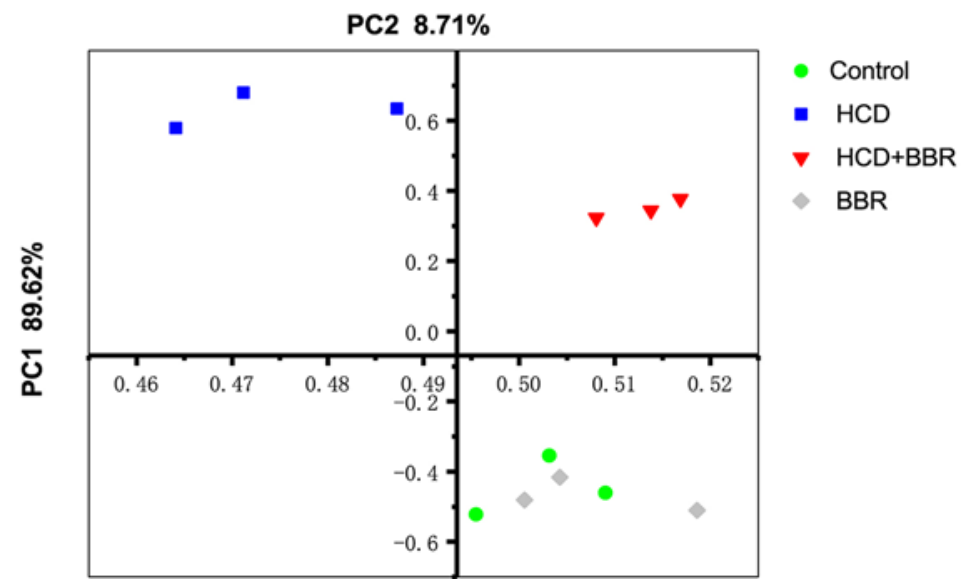

FIGURE 3 | Raman spectral analysis of liver tissue of zebrafish larvae treated with berberine (BBR). (A) Raman spectra obtained from the liver of zebrafish with different treatments. (B) Principal component analysis (PCA) of the spectra extracted from the Raman spectral datasets of the control, BRR(5 $\mu \mathrm{M}), \mathrm{HCD}$, and HCD +BBR $(5 \mu \mathrm{M})$. Each liver of zebrafish was determined under the microscope at least three different regions for at least three times to acquire the average spectrum. 
A
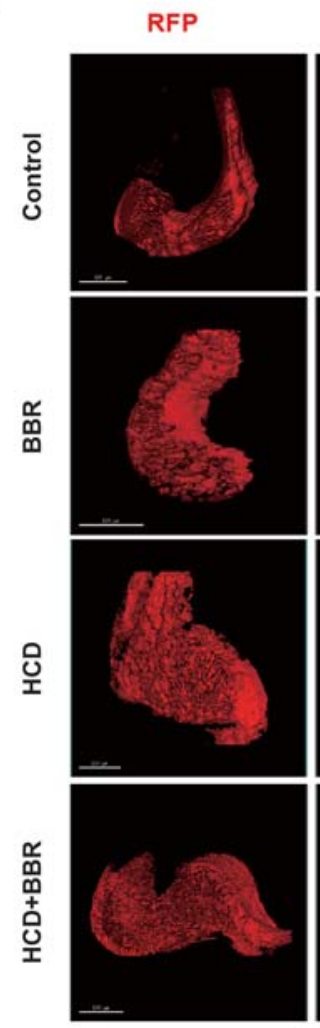

ROS
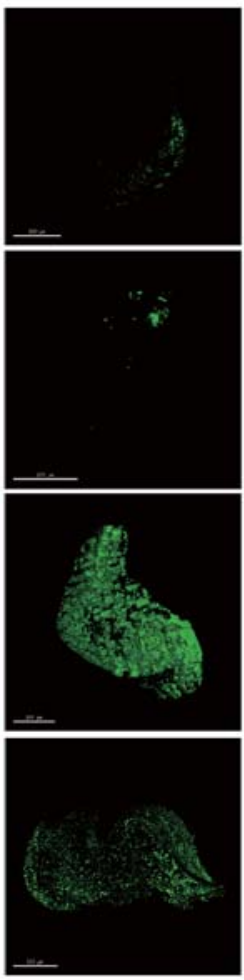
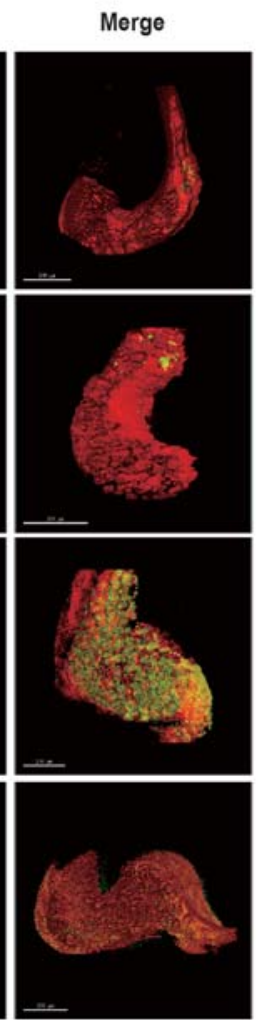

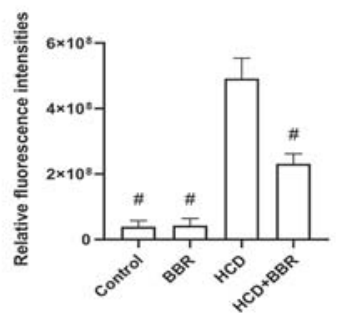

C
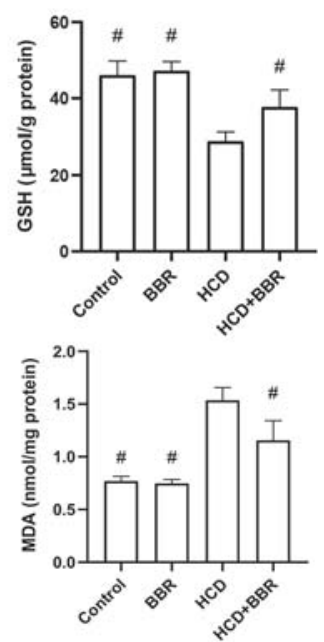

FIGURE 4 | Effects of berberine (BBR) on the hepatic oxidative stress in high-cholesterol diet (HCD)-induced zebrafish larvae. (A) Liver volume rendering of reactive oxygen species (ROS) staining showed that BBR (5 $\mu \mathrm{M})$ attenuated hepatic ROS level in HCD-induced Tg (fabp10a: dsRed) zebrafish. ROS generation in the Tg (fabp10a: dsRed) zebrafish liver treated with HCD. Hepatic ROS were detected with CellROX ${ }^{\circledR}$ Green Reagent. Bar $=100 \mu \mathrm{m}$. (B) Quantitative analysis of ROS throughout the entire zebrafish liver. Effects of BBR $(5 \mu \mathrm{M})$ on the glutathione (GSH) (C) and malondialdehyde (MDA) (D) levels in HCD-induced zebrafish larvae. "Compared with the HCD group, $P<0.05$.

BBR group (Figure 5B). Of these genes, 172 had increased expression levels and 296 had significantly decreased expression levels (Figures 5C, D).

\section{KEGG Pathway and GO Enrichment Analysis Based on Combined DEGs}

To figure out the possible mechanism of BBR on the zebrafish NAFLD model, we conducted a function-annotations analysis from the co-DEGs (Ctrl vs. M combined BBR vs. M). The coupregulation and co-downregulation in DEGs were enriched into the GO biological process, as shown in Figures $6 \mathbf{A}, \mathbf{B}$, respectively. We further applied the KEGG ontology to classify the functional annotation of genes. A total of 124 genes can be assigned to the 10 pathways. As expected, most genes were classified into the metabolic pathways, such as PPAR signaling pathway, glycolysis/gluconeogenesis, glycerophospholipid metabolism, glycerolipid metabolism, and pyrimidine metabolism. We noted that ggt1a, ggt 1l2.2, gpx7, and rrm2 genes were classified into glutathione metabolism term, which may partially explain the antioxidant mechanism of BBR. The genepathway interaction network was shown in Figure 6C. Functional category analysis by DAVID included lipid metabolism, DNA damage and repair, and iron, which was closely associated with pediatric NAFLD (Feldman et al., 2015; Mann et al., 2017; Baker and Friedman, 2018). Several vital genes involved in these pathways were enriched, including ch25h1.1, ch25h, fads2, lpl, mvda, plcg1, h2afx, parpbp, pif1, rev1, primpol, ube2t, tk1, cyp $2 \times 12$, cyp51, cyp46a1.1, jmjd6, and iscal (Figure 6D). Excessive triglyceride accumulation in the hepatocytes causes oxidative stress, lipotoxicity, and subsequent DNA damage (Mann et al., 2017). These results indicated that the protective effects of BBR on hepatocytes might be related to the improvement of lipid metabolism, oxidative stress, and iron homeostasis.

\section{Validation of Microarray Analysis Genes by qRT-PCR}

To confirm the DEGs detected with microarray analysis, we selected the significant enrichment genes belonging to lipid metabolism, DNA damage and repair, and iron of which have the homologous gene in human for transcriptional validation (as shown in Figure 7). Results showed that BBR $(5 \mu \mathrm{M})$ significantly decreased the gene expression, such as ch25, fads2, msmo1, mvda, plcg1, h2afx, parpbp, pif1, primpol, ube2t, and $t k 1$, and increased the gene expression of $l p l$, revl, jmjd6, and iscal (Figure 7). These gene expressions detected by qRT-PCR were in good agreement with those by microarray analysis. 
A

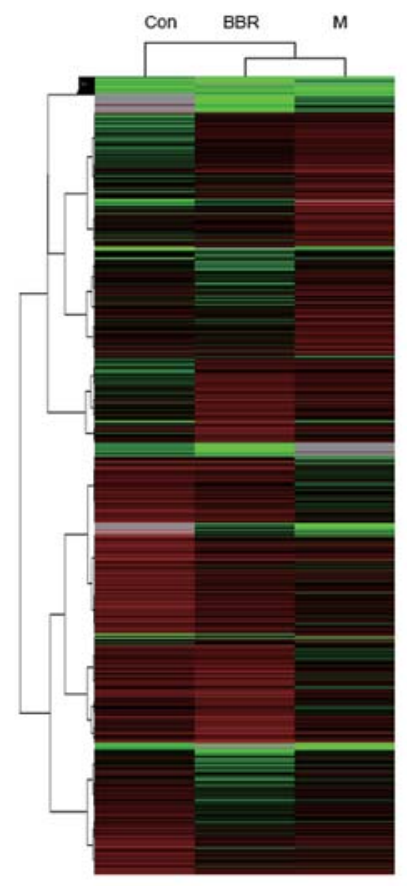

C

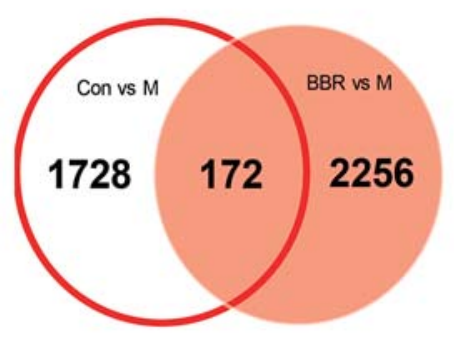

D
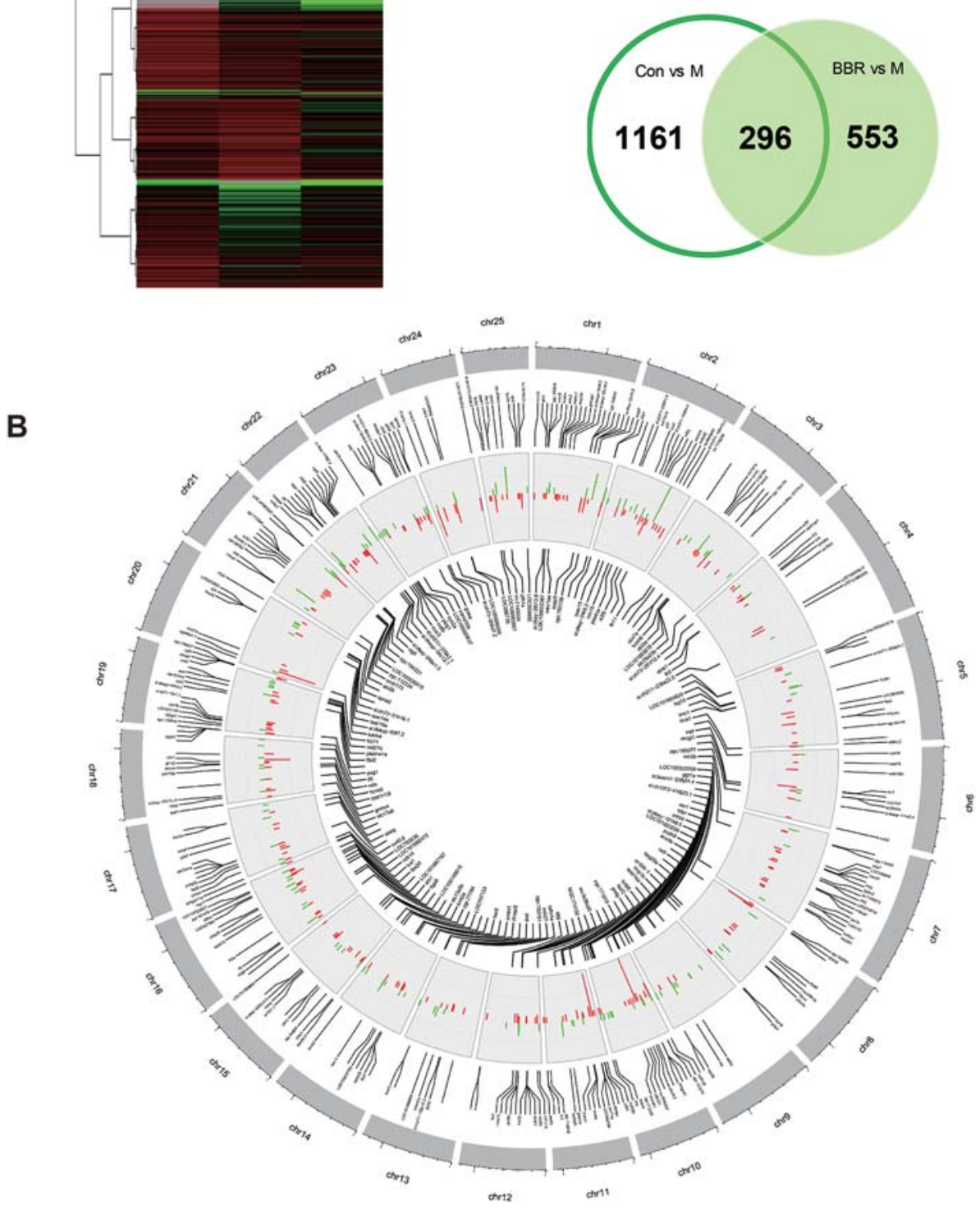

FIGURE 5 | Trend analysis of gene expression patterns in larval zebrafish liver. (A) Cluster analysis of genes and samples from differentially expressed genes (DEGs). (B) The common DEGs in control and BBR groups compared with the HCD group shown by Circos plot. Red color, upregulated genes; green color, downregulated genes. (C) Comparison of upregulated DEGs between different groups. (D) Comparison of downregulated DEGs between different groups. 


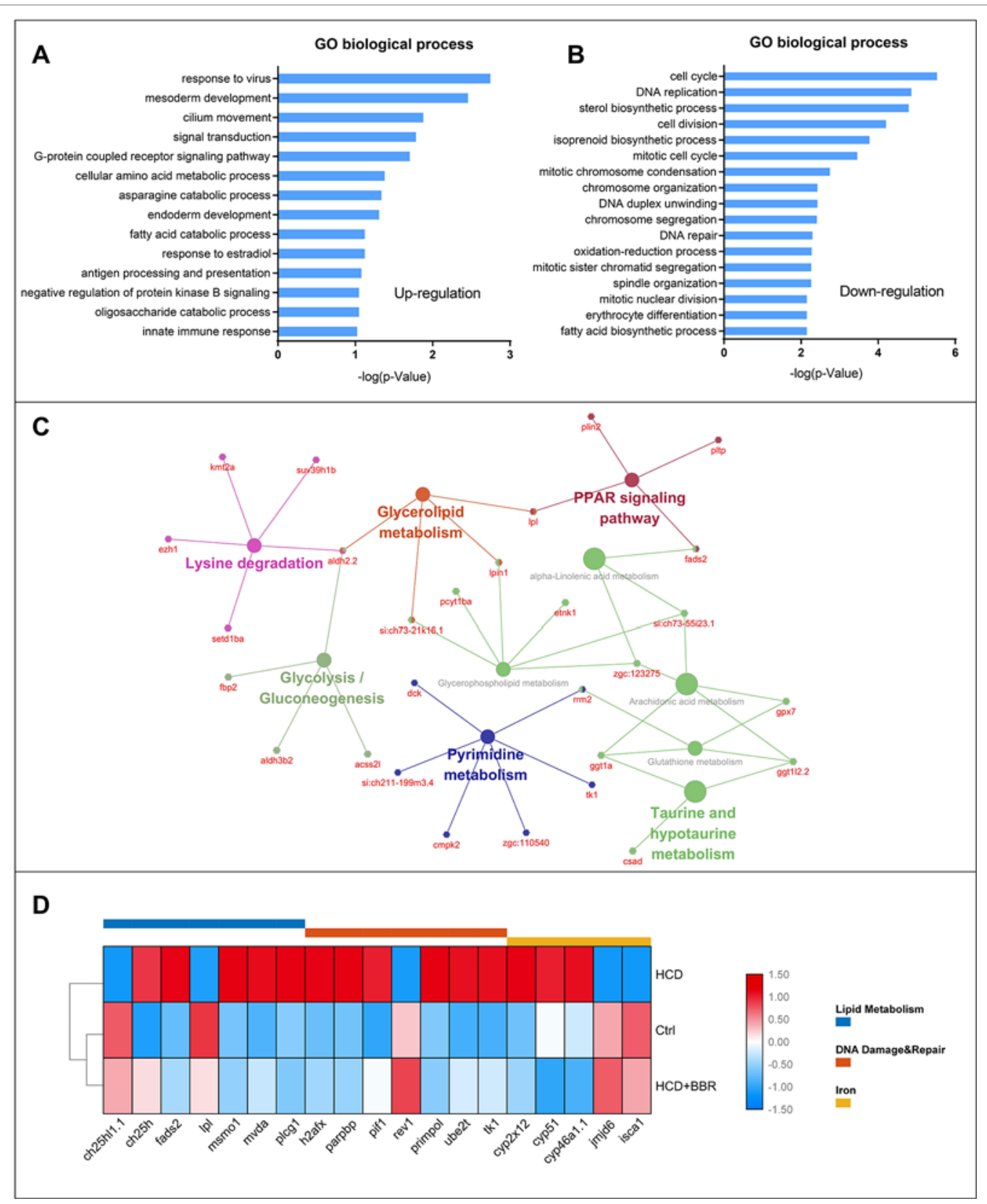

FIGURE 6 | Gene ontology, KEGG enrichment, and functional category analysis. (A) Gene ontology from the upregulated differentially expressed genes (DEGs) (Ctrl vs. M combined $5 \mu \mathrm{M}$ BBR vs. M). (B) Gene ontology from the downregulated DEGs (Ctrl vs. M combined $5 \mu \mathrm{M}$ BBR vs. M). (C) KEGG pathway enrichment results for the total DEGs. (D) Gene collection from functional category analysis by DAVID.

\section{DISCUSSION}

NAFLD was commonly considered a disease of adults. However, accumulative evidence showed that the prevalence of NAFLD rise with the growing epidemic in the pediatric population around the world (Welsh et al., 2013). Several reports revealed that there is a heterogeneity in the patterns of the histologic lesions between pediatric and adult NAFLD/NASH (Crespo et al., 2016). This suggests that the pathophysiology of NAFLD may have significant differences between the adult and pediatric types (Papandreou et al., 2007). The pathogenesis of [ediatric NAFLD is still not fully understood. The scientific evidence indicates that a genetic predisposition interplaying with unhealthy diets and lifestyles might influence the occurrence and development of NAFLD in the pediatric population, mearly $50 \%$ of overweight children and adolescents accompanied by NAFLD (Betancourt-Garcia et al., 2017). In addition, insulin resistance and increased hepatic glucose production affect the 

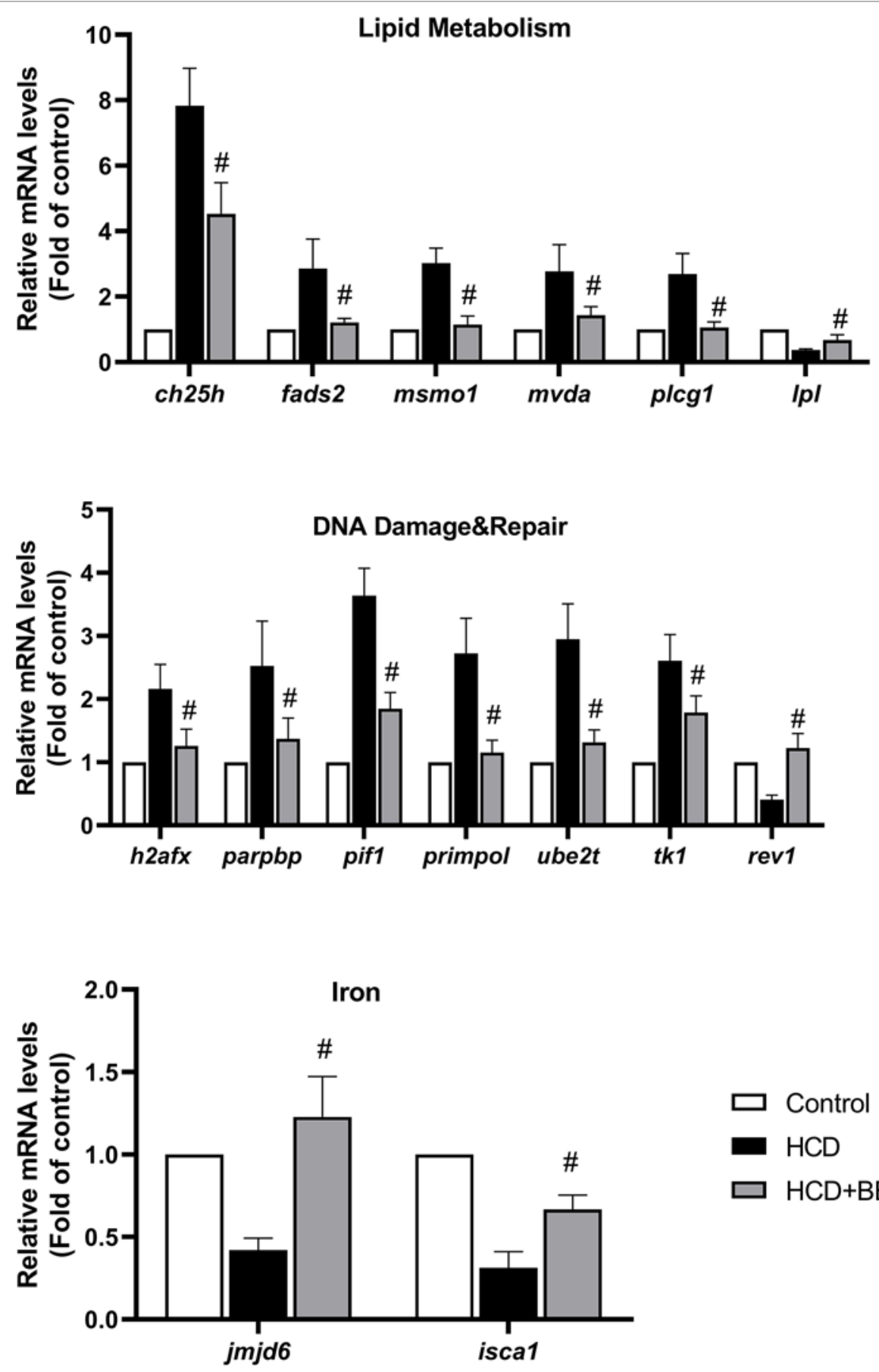

FIGURE 7 | Validation of the microarray data using quantitative real-time PCR (qRT-PCR). Relative expression levels of the selected genes determined using qRTPCR. "Compared with the HCD group, $P<0.05$.

hepatic de novo lipogenesis (Ciba and Widhalm, 2007). The knowledge gap of the pathogenesis of NAFLD/NASH in children is partially due to a lack of studies in animal models that may resemble pediatric NAFLD (Patton et al., 2006; Papandreou et al., 2007). In recent years, zebrafish have been gaining popularity in lipid research (Holtta-Vuori et al., 2010). We previously established a diet-induced steatosis model in larval zebrafish to mimic the progression of hepatic steatosis of the pediatric population and assess anti-steatosis agents (Chen et al., 2018). Given the transparent feature of larval zebrafish, we introduced Raman imaging and 3D molecular imaging to detect the change of the biochemistry composition and oxidative stress in zebrafish liver. These approaches could also be applied to other pharmacology and biochemistry study. 
Several studies have reported that BBR exerts beneficial effects on NAFLD in adult patients and animal models through various potential mechanisms such as improvement of insulin sensitivity, adenosine monophosphate-activated protein kinase (AMPK) pathway, mitochondrial function, oxidative stress, and gut microenvironment (Yan et al., 2015; Zhu et al., 2016). The pediatric population is supposedly more susceptible to drug interventions (Hatton et al., 2018). Moreover, its availability and pharmacology mechanism may not be entirely consistent with adults (Hatton et al., 2018). Thus, we employed a diet-induced larval zebrafish model. Our results showed that BBR could significantly reduce the BMI, hepatic cholesterol, and triglyceride and improve hepatic steatosis in high-fat diet-treated zebrafish. These results suggest that berberine exerts its anti-steatosis through moderating the lipid metabolism and are similar to those obtained in adult rodent model studies (Zhao et al., 2017; Deng et al., 2019; Luo et al., 2019; Zhang et al., 2019). In addition, recent studies revealed that BBR could regulate adipose-mediated energy expenditure, which may exert an indirect effect on liver steatosis and further explain the reason for the reduction in body weight and BMI after berberine treatment in this study (Sun et al., 2018; Wu et al., 2019). Also, Raman mapping indicated that BBR treatment did not affect the hepatic biochemical composition in the healthy larvae. This evidence provides a new clue that BBR has the potential to prevent hepatic steatosis for the pediatric population.

Microarray is a high-throughput and rapid technique relative to traditional methods to detect transcriptome information. Accordingly, we investigated the transcriptome responses of zebrafish liver to BBR treatment using microarray. The transcriptome information of zebrafish liver under BBR treatment in the NAFLD model was presented for the first time and could provide the molecular basis for the pharmacological mechanism of BBR to larval zebrafish. GO analysis of the genes with differentially upregulated expression in the BBR group indicated that some genes were associated with the categories of response to virus (GO:0009615), antigen processing and presentation (GO:0019882), and innate immune response (GO:0045087). In addition to genetic and environmental factors on liver disease, increasing evidence showed that gut microbial composition has a strong influence on the pathogenesis of NAFLD (Bashiardes et al., 2016). Childhood allergic diseases exhibit high correlation to the early gut microbiota system in response to the immunity of infancy (Jenmalm, 2017). The changes in these associated immune genes might be supposed to high-fat diet challenge and BBR treatment. It has been reported that BBR could modulate hepatic lipid metabolism and exert hepatic protection by improving gut homeostasis (Sun et al., 2017; Qin et al., 2018; Shi et al., 2018). Thus, this may be a key mechanism of $\mathrm{BBR}$ in pediatric NAFLD treatment.

Moreover, GO term from differentially downregulated expression in the BBR group demonstrated that BBR might attenuate the fatty acid biosynthetic process, sterol biosynthetic process, and oxidation stress. KEGG pathway analysis suggested that the differentially expressed genes in the BBR group are mainly involved in the metabolic pathways, which are similar to previous reports (Qiang et al., 2016; Zhao et al., 2017; Deng et al., 2019). By functional annotation tool DAVID, we identified several essential genes involved in lipid metabolism, DNA damage and repair, and iron. Notably, studies showed that the prevalence of iron deficiency was higher in obese children than normal-weight subjects (Nead et al., 2004; Cepeda-Lopez et al., 2011). Moreover, children with NAFLD have higher serum levels of hepcidin than those without NAFLD (Amato et al., 2010). NAFLD may impact on iron homeostasis through a different mechanism from mere obesity-induced iron deficiency (Feldman et al., 2015). The regulation of iron metabolism by BBR needs further study and may become a potential approach for pediatric NAFLD. Besides, BBR has no effect on the genes related to liver lipogenesis (srebp1, fas, acc1, acc2, and $s c d 1$ ), fatty acid betaoxidation (ppara and cpt1a), and VLDL secretion ( $m t p)$ in this study (Figure S2).

In recent years, Raman microspectroscopy has emerged as an attractive diagnostic tool (Zhu et al., 2016). Raman microspectroscopy provides a biochemical map of interested tissue that potentially enables the identification of spatialtemporal changes in molecular composition. Several studies reported the use of confocal Raman imaging for the detection of NAFLD (Hewitt et al., 2015; Yan et al., 2017). Raman imaging allows label-free approaches to observe and quantify the lipid accumulation within the liver without the need for contrast agents (Yan et al., 2017). Unlike rodent animal models, the biochemistry analysis of larval zebrafish liver is a challenge since the tiny size of the larval zebrafish liver is difficult to handle. We thus introduced Raman confocal microspectroscopy to analyze the changes of biochemistry component in the larval liver. Besides the differences in the lipid component, we also found an increase of DNA in the liver in HCD-treated zebrafish. Of note, NAFLD can ultimately lead to the development of hepatocellular cancer in some patients (Rinella, 2015). The increase in DNA may be indicative of malignancy (Cai et al., 2017). Particularly, BBR treatment could drop the peaks of DNA and lipid/protein, suggesting that it may delay the development of progressive NAFLD.

The pathogenesis of NAFLD and NASH remains poorly understood. The progression of NAFLD to NASH has been explained by the "two-hit" theory (Wruck et al., 2017). The "first hit" involves the accumulation of fat in the liver (Day and James, 1998). Oxidative stress and induction of adipocytokine are thought to play a role in the second "hit" (Spahis et al., 2017). It is convinced that NAFLD is closely associated with oxidative stress (Spahis et al., 2017). Excess lipids impact metabolic pathways, increase ROS generation, and damage to mitochondrial DNA (Dasarathy et al., 2011). TEM image showed that BBR corrected the abnormal structure of the mitochondria. Raman analysis suggested that BBR could affect DNA content. Molecular imaging demonstrated that BBR could eliminate hepatic ROS in the larval zebrafish model. By comprehensive analysis of those results, it indicated that BBR might alleviate excess ROS generation in the HCD-induced 
zebrafish model through improving mitochondrial quality. Cysteamine could reduce ROS by restoring glutathione levels (Caccamo et al., 2005). It acts as a novel therapeutic agent in nonalcoholic steatohepatitis (NASH), which has recently been used in a phase IIb trial in children with NASH (Schwimmer et al., 2016). The above evidence implies that BBR has the potential to treat pediatric NAFLD.

In summary, we first employed a larval zebrafish model to assess the effects and possible mechanism of BBR in the treatment of hepatic steatosis. Several genes involved in lipid metabolism, DNA damage and repair, and iron have been identified by microarray analysis. These results might provide molecular information that will facilitate understanding of BBR on the treatment of pediatric NAFLD. Also, further studies are required to validate and extend the findings of the results in this study. In research methods, based on the advantage of zebrafish, we introduced Raman imaging and 3D molecular imaging to analyze the biochemistry component and ROS level in the liver of zebrafish, respectively. Our study offers a new idea and extends the application of zebrafish models.

\section{DATA AVAILABILITY STATEMENT}

The datasets generated for this study can be found in the GEO repository, GSE136436, https://www.ncbi.nlm.nih.gov/geo/ query/acc.cgi? acc=GSE136436.

\section{ETHICS STATEMENT}

The animal study was reviewed and approved by the Laboratory Animal Management and Animal Welfare Committee in the

\section{REFERENCES}

Albadri, S., De Santis, F., Di Donato, V., and Del Bene, F. (2017). CRISPR/ Cas9-Mediated Knockin and Knockout in Zebrafish. Genome Editing in Neurosciences. Eds. R. Jaenisch, F. Zhang and F. Gage [Cham (CH): Genome Editing in Neurosciences], 41-49.

Amato, A., Santoro, N., Calabro, P., Grandone, A., Swinkels, D. W., Perrone, L., et al. (2010). Effect of body mass index reduction on serum hepcidin levels and iron status in obese children. Int. J. Obes (Lond) 34 (12), 1772-1774. doi: 10.1038/ijo.2010.204

Assuncao, S. N. F., Sorte, N. C. B., Alves, C. D., Mendes, P. S. A., Alves, C. R. B., and Silva, L. R. (2017). Nonalcoholic fatty liver disease (NAFLD) pathophysiology in obese children and adolescents: update. Nutr. Hosp. 34 (3), 727-730. doi: 10.20960/nh.723

Baker, P. R., and Friedman, J. E. (2018). Mitochondrial role in the neonatal predisposition to developing nonalcoholic fatty liver disease. J. Clin. Invest. 128 (9), 3692-3703. doi: 10.1172/JCI120846

Barros, T. P., Alderton, W. K., Reynolds, H. M., Roach, A. G., and Berghmans, S. (2008). Zebrafish: an emerging technology forin vivo pharmacological assessment to identify potential safety liabilities in early drug discovery. Br. J. Pharmacol. 1547, 1400-1413. doi: 10.1038/bjp.2008.249

Bashiardes, S., Shapiro, H., Rozin, S., Shibolet, O., and Elinav, E. (2016). Nonalcoholic fatty liver and the gut microbiota. Mol. Metab. 5 (9), 782-794. doi: 10.1016/j.molmet.2016.06.003

Betancourt-Garcia, M. M., Arguelles, A., Montes, J., Hernandez, A., Singh, M., and Forse, R. A. (2017). Pediatric nonalcoholic fatty liver disease: the rise of a lethal
Institute of Medicinal Biotechnology, Chinese Academy of Medical Sciences.

\section{AUTHOR CONTRIBUTIONS}

$\mathrm{J}-\mathrm{PZ}$ conceived and designed this project. $\mathrm{BC}$ and $\mathrm{J}-\mathrm{PZ}$ wrote the manuscript. C-QH reviewed the manuscript. BC performed the bioinformatics analysis and biochemistry experiment. $\mathrm{Y}-\mathrm{MZ}$ and YH performed the Raman spectroscopy experiments, M-QZ performed the PCR experiment.

\section{FUNDING}

This work was supported by the CAMS Initiative for Innovative Medicine (grant number 2016-I2M-1-011), by Foundation for Innovative Research Groups of the National Natural Science Foundation of China (grant number 81621064), and by the National Natural Science Foundation of China (grant number 81603172).

\section{ACKNOWLEDGMENTS}

We thank Jie Meng for the fish husbandry.

\section{SUPPLEMENTARY MATERIAL}

The Supplementary Material for this article can be found online at: https://www.frontiersin.org/articles/10.3389/fphar.2019. 01504/full\#supplementary-material

disease among Mexican American hispanic children. Obes. Surg. 27 (1), 236244. doi: 10.1007/s11695-016-2440-5

Caccamo, D., Campisi, A., Curro, M., Bramanti, V., Tringali, M., Li Volti, G., et al. (2005). Antioxidant treatment inhibited glutamate-evoked NF-kappaB activation in primary astroglial cell cultures. Neurotoxicology 26 (5), 915921. doi: 10.1016/j.neuro.2005.01.010

Cai, Z. X., Chen, G., Zeng, Y. Y., Dong, X. Q., Lin, M. J., Huang, X. H., et al. (2017). Circulating tumor DNA profiling reveals clonal evolution and real-time disease progression in advanced hepatocellular carcinoma. Int. J. Cancer 141 (5), $977-$ 985. doi: 10.1002/ijc.30798

Cepeda-Lopez, A. C., Osendarp, S. J., Melse-Boonstra, A., Aeberli, I., GonzalezSalazar, F., Feskens, E., et al. (2011). Sharply higher rates of iron deficiency in obese Mexican women and children are predicted by obesity-related inflammation rather than by differences in dietary iron intake. Am. J. Clin. Nutr. 93 (5), 975-983. doi: 10.3945/ajcn.110.005439

Chakrova, N., Rieger, B., and Stallinga, S. (2016). Deconvolution methods for structured illumination microscopy. J. Opt Soc. Am. A Opt. Image Sci. Vis. 33 (7), B12-B20. doi: 10.1364/JOSAA.33.000B12

Chen, B., Gao, Z. Q., Liu, Y., Zheng, Y. M., Han, Y., Zhang, J. P., et al. (2017). Embryo and developmental toxicity of cefazolin sodium impurities in zebrafish. Front. Pharmacol. 8, 403. doi: 10.3389/fphar.2017.00403

Chen, B., Zheng, Y. M., and Zhang, J. P. (2018). Comparative study of different diets-induced NAFLD models of Zebrafish. Front. Endocrinol. >(Lausanne) 9, 366. doi: $10.3389 /$ fendo. 2018.00366

Ciba, I., and Widhalm, K. (2007). The association between non-alcoholic fatty liver disease and insulin resistance in 20 obese children and 
adolescents. Acta Paediatr. 96 (1), 109-112. doi: 10.1111/j.1651-2227.2007. 00031.x

Crespo, M., Lappe, S., Feldstein, A. E., and Alkhouri, N. (2016). Similarities and differences between pediatric and adult nonalcoholic fatty liver disease. Metabolism 65 (8), 1161-1171. doi: 10.1016/j.metabol.2016.01.008

Dai, W., Wang, K., Zheng, X., Chen, X., Zhang, W., Zhang, Y., et al. (2015). High fat plus high cholesterol diet lead to hepatic steatosis in zebrafish larvae: a novel model for screening anti-hepatic steatosis drugs. Nutr. Metab. (Lond) 12, 42. doi: 10.1186/s12986-015-0036-Z

Dasarathy, S., Yang, Y., McCullough, A. J., Marczewski, S., Bennett, C., and Kalhan, S. C. (2011). Elevated hepatic fatty acid oxidation, high plasma fibroblast growth factor 21, and fasting bile acids in nonalcoholic steatohepatitis. Eur. J. Gastroenterol. Hepatol 23 (5), 382-388. doi: 10.1097/MEG.0b013e328345c8c7

Day, C. P., and James, O. F. (1998). Steatohepatitis: a tale of two "hits"? Gastroenterology 114 (4), 842-845. doi: 10.1016/s0016-5085(98)70599-2

Day, C. P. (2006). From fat to inflammation. Gastroenterology 130 (1), 207-210. doi: 10.1053/j.gastro.2005.11.017

Deng, Y., Tang, K., Chen, R., Nie, H., Liang, S., Zhang, J., et al. (2019). Berberine attenuates hepatic oxidative stress in rats with non-alcoholic fatty liver disease via the Nrf2/ARE signalling pathway. Exp. Ther. Med. 17 (3), 2091-208s.doi: 10.3892/etm.2019.7208

EauClaire, S. F., Cui, S., Ma, L., Matous, J., Marlow, F. L., Gupta, T., et al. (2012). Mutations in vacuolar H+ -ATPase subunits lead to biliary developmental defects in zebrafish. Dev. Biol. 365 (2), 434-444. doi: 10.1016/ j.ydbio.2012.03.009

Feldman, A., Aigner, E., Weghuber, D., and Paulmichl, K. (2015). The potential role of iron and copper in pediatric obesity and nonalcoholic fatty liver disease. BioMed. Res. Int. 2015, 287401. doi: 10.1155/2015/287401

Gu, Q., Yang, X., Lin, L., Li, S., Li, Q., Zhong, S., et al. (2014). Genetic ablation of solute carrier family 7a3a leads to hepatic steatosis in zebrafish during fasting. Hepatology 60 (6), 1929-1941. doi: 10.1002/hep.27356

Han, Y., Chen, B., Zhang, J., and Hu, C. (2018). Cardiac safety evaluation in zebrafish and in silico ADME prediction of cephalosporins with an aminothiazoyl ring at the C-7 position. Toxicol. Appl. Pharmacol. 347, 3344. doi: 10.1016/j.taap.2018.03.022

Hatton, G., Alterio, T., Nobili, V., and Mann, J. P. (2018). Unmet needs in pediatric NAFLD research: what do we need to prioritize for the future? Expert Rev. Gastroenterol. Hepatol 12 (10), 961-967. doi: 10.1080/ 17474124.2018.1512853

Hewitt, K. C., Ghassemi Rad, J., McGregor, H. C., Brouwers, E., Sapp, H., Short, M. A., et al. (2015). Accurate assessment of liver steatosis in animal models using a high throughput Raman fiber optic probe. Analyst 140 (19), 6602-6609. doi: 10.1039/c5an01080b

Holtta-Vuori, M., Salo, V. T., Nyberg, L., Brackmann, C., Enejder, A., Panula, P., et al. (2010). Zebrafish: gaining popularity in lipid research. Biochem. J. 429 (2), 235-242. doi: 10.1042/BJ20100293

Hu, Z., Zhang, J., and Zhang, Q. (2011). Expression pattern and functions of autophagy-related gene atg5 in zebrafish organogenesis. Autophagy 7 (12), 1514-1527. doi: 10.4161/auto.7.12.18040

Imenshahidi, M., and Hosseinzadeh, H. (2019). Berberine and barberry (Berberis vulgaris): a clinical review. Phytother. Res. 33 (3), 504-523. doi: 10.1002/ ptr.6252

Jenmalm, M. C. (2017). The mother-offspring dyad: microbial transmission, immune interactions and allergy development. J. Intern. Med. 282 (6), 484495. doi: 10.1111 /joim. 12652

Kanehisa, M., and Goto, S. (2000). KEGG: kyoto encyclopedia of genes and genomes. Nucleic Acids Res. 28 (1), 27-30. doi: 10.1093/nar/28.1.27

Lohi, O., Parikka, M., and Ramet, M. (2013). The zebrafish as a model for paediatric diseases. Acta Paediatr. 102 (2), 104-110. doi: 10.1111/j.16512227.2012.02835.x

Luo, Y., Tian, G., Zhuang, Z., Chen, J., You, N., Zhuo, L., et al. (2019). Berberine prevents non-alcoholic steatohepatitis-derived hepatocellular carcinoma by inhibiting inflammation and angiogenesis in mice. Am. J. Trans. Res. 11 (5), 2668-2682.

Mahfood Haddad, T., Hamdeh, S., Kanmanthareddy, A., and Alla, V. M. (2017). Nonalcoholic fatty liver disease and the risk of clinical cardiovascular events: a systematic review and meta-analysis. Diabetes Metab. Syndr. 11 (Supplement 1), S209-S216. doi: 10.1016/j.dsx.2016.12.033
Majzner, K., Kochan, K., Kachamakova-Trojanowska, N., Maslak, E., Chlopicki, S., and Baranska, M. (2014). Raman imaging providing insights into chemical composition of lipid droplets of different size and origin: in hepatocytes and endothelium. Anal. Chem. 86 (13), 6666-6674. doi: 10.1021/ac501395g

Mann, J. P., Raponi, M., and Nobili, V. (2017). Clinical implications of understanding the association between oxidative stress and pediatric NAFLD. Expert Rev. Gastroenterol. Hepatol 11 (4), 371-382. doi: 10.1080/ 17474124.2017.1291340

Nead, K. G., Halterman, J. S., Kaczorowski, J. M., Auinger, P., and Weitzman, M. (2004). Overweight children and adolescents: a risk group for iron deficiency. Pediatrics 114 (1), 104-108. doi: 10.1542/peds.114.1.104

Nobili, V., Marcellini, M., Devito, R., Ciampalini, P., Piemonte, F., Comparcola, D., et al. (2006). NAFLD in children: a prospective clinical-pathological study and effect of lifestyle advice. Hepatology 44 (2), 458-465. doi: 10.1002/hep.21262

Nobili, V., Alisi, A., Newton, K. P., and Schwimmer, J. B. (2016). Comparison of the phenotype and approach to pediatric vs adult patients with nonalcoholic fatty liver disease. Gastroenterology 150 (8), 1798-1810. doi: 10.1053/ j.gastro.2016.03.009

Papandreou, D., Rousso, I., and Mavromichalis, I. (2007). Update on nonalcoholic fatty liver disease in children. Clin. Nutr. 26 (4), 409-415. doi: 10.1016/j.clnu.2007.02.002

Paradies, G., Paradies, V., Ruggiero, F. M., and Petrosillo, G. (2014). Oxidative stress, cardiolipin and mitochondrial dysfunction in nonalcoholic fatty liver disease. World J. Gastroenterol. 20 (39), 14205-14218. doi: 10.3748/ wjg.v20.i39.14205

Patton, H. M., Sirlin, C., Behling, C., Middleton, M., Schwimmer, J. B., and Lavine, J. E. (2006). Pediatric nonalcoholic fatty liver disease: a critical appraisal of current data and implications for future research. J. Pediatr. Gastroenterol. Nutr. 43 (4), 413-427. doi: 10.1097/01.mpg.0000239995.58388.56

Pegard, N. C., and Fleischer, J. W. (2013). Three-dimensional deconvolution microfluidic microscopy using a tilted channel. J. Biomed. optics 18 (4), 040503. doi: $10.1117 / 1$.JBO.18.4.040503

Qiang, X., Xu, L., Zhang, M., Zhang, P., Wang, Y., Wang, Y., et al. (2016) Demethyleneberberine attenuates non-alcoholic fatty liver disease with activation of AMPK and inhibition of oxidative stress. Biochem. Biophys. Res. Commun. 472 (4), 603-609. doi: 10.1016/j.bbrc.2016.03.019

Qin, C., Zhang, H., Zhao, L., Zeng, M., Huang, W., Fu, G., et al. (2018). Microbiota transplantation reveals beneficial impact of berberine on hepatotoxicity by improving gut homeostasis. Sci. China Life Sci. 61 (12), 1537-1544. doi: 10.1007/s11427-017-9202-0

Richter, K., and Kietzmann, T. (2016). Reactive oxygen species and fibrosis: further evidence of a significant liaison. Cell Tissue Res. 365 (3), 591-605. doi: $10.1007 / \mathrm{s} 00441-016-2445-3$

Rinella, M. E. (2015). Nonalcoholic fatty liver disease: a systematic review. JAMA 313 (22), 2263-2273. doi: 10.1001/jama.2015.5370

Sapp, V., Gaffney, L., EauClaire, S. F., and Matthews, R. P. (2014). Fructose leads to hepatic steatosis in zebrafish that is reversed by mechanistic target of rapamycin (mTOR) inhibition. Hepatology 60 (5), 1581-1592. doi: 10.1002/ hep. 27284

Schlegel, A. (2012). Studying non-alcoholic fatty liver disease with zebrafish: a confluence of optics, genetics, and physiology. Cell Mol. Life Sci. 69 (23), 39533961. doi: 10.1007/s00018-012-1037-y

Schwimmer, J. B., Lavine, J. E., Wilson, L. A., Neuschwander-Tetri, B. A., Xanthakos, S. A., Kohli, R., et al. (2016). Children with nonalcoholic fatty liver disease, cysteamine bitartrate delayed release improves liver enzymes but does not reduce disease activity scores. Gastroenterology 151 (6), 1141-54 e9. doi: 10.1053/j.gastro.2016.08.027

Shetty, G., Kendall, C., Shepherd, N., Stone, N., and Barr, H. (2006). Raman spectroscopy: elucidation of biochemical changes in carcinogenesis of oesophagus. Br. J. Cancer 94 (10), 1460-1464. doi: 10.1038/sj.bjc.6603102

Shi, Y., Hu, J., Geng, J., Hu, T., Wang, B., Yan, W., et al. (2018). Berberine treatment reduces atherosclerosis by mediating gut microbiota in apoE-/- mice. BioMed. Pharmacother. 107, 1556-1563. doi: 10.1016/j.biopha.2018.08.148

Spahis, S., Delvin, E., Borys, J. M., and Levy, E. (2017). Oxidative stress as a critical factor in nonalcoholic fatty liver disease pathogenesis. Antioxidants Redox Signaling 26 (10), 519-541. doi: 10.1089/ars.2016.6776

Stoletov, K., Fang, L., Choi, S. H., Hartvigsen, K., Hansen, L. F., Hall, C., et al. (2009). Vascular lipid accumulation, lipoprotein oxidation, and macrophage 
lipid uptake in hypercholesterolemic zebrafish. Circ. Res. 104 (8), 952-960. doi: 10.1161/CIRCRESAHA.108.189803

Sun, R., Yang, N., Kong, B., Cao, B., Feng, D., Yu, X., et al. (2017). Orally administered berberine modulates hepatic lipid metabolism by altering microbial bile acid metabolism and the intestinal FXR signaling pathway. Mol. Pharmacol. 91 (2), 110-122. doi: 10.1124/mol.116.106617

Sun, Y., Xia, M., Yan, H., Han, Y., Zhang, F., Hu, Z., et al. (2018). Berberine attenuates hepatic steatosis and enhances energy expenditure in mice by inducing autophagy and fibroblast growth factor 21. Br. J. Pharmacol. 175 (2), 374-387. doi: 10.1111/bph.14079

Veldman, M. B., and Lin, S. (2008). Zebrafish as a developmental model organism for pediatric research. Pediatr. Res. 64 (5), 470-476. doi: 10.1203/PDR.0b013 e318186e609

Welsh, J. A., Karpen, S., and Vos, M. B. (2013). Increasing prevalence of nonalcoholic fatty liver disease among United States adolescents, 1988-1994 to 2007-2010. J. Pediatr. 162 (3), 496-500. doi: 10.1016/j.jpeds.2012.08.043

Wruck, W., Graffmann, N., Kawala, M. A., and Adjaye, J. (2017). Concise review: current status and future directions on research related to nonalcoholic fatty liver disease. Stem Cells 35 (1), 89-96. doi: 10.1002/stem.2454

Wu, L., Xia, M., Duan, Y., Zhang, L., Jiang, H., Hu, X., et al. (2019). Berberine promotes the recruitment and activation of brown adipose tissue in mice and humans. Cell Death Dis. 10 (6), 468. doi: 10.1038/s41419-019-1706-y

Yan, H. M., Xia, M. F., Wang, Y., Chang, X. X., Yao, X. Z., Rao, S. X., et al. (2015). Efficacy of berberine in patients with non-alcoholic fatty liver disease. PloS One 10 (8), e0134172. doi: 10.1371/journal.pone.0134172

Yan, J., Yu, Y., Kang, J. W., Tam, Z. Y., Xu, S., Fong, E. L. S., et al. (2017). Development of a classification model for non-alcoholic steatohepatitis (NASH) using confocal Raman micro-spectroscopy. J. Biophotonics 10 (12), 1703-1713. doi: 10.1002/jbio.201600303
Yu, Y., Ouyang, Y., and Yao, W. (2018). shinyCircos: an R/Shiny application for interactive creation of Circos plot. Bioinformatics 34 (7), 1229-1231. doi: 10.1093/bioinformatics/btx763

Zhang, Y. P., Deng, Y. J., Tang, K. R., Chen, R. S., Liang, S., Liang, Y. J., et al. (2019). Berberine ameliorates high-fat diet-induced non-alcoholic fatty liver disease in rats via activation of SIRT3/AMPK/ACC pathway. Curr. Med. Sci. 39 (1), 37-43.doi: 10.1007/s11596-019-1997-3

Zhao, L., Cang, Z., Sun, H., Nie, X., Wang, N., and Lu, Y. (2017). Berberine improves glucogenesis and lipid metabolism in nonalcoholic fatty liver disease. BMC Endocr. Disord. 17 (1), 13. doi: 10.1186/s12902-017-0165-7

Zheng, Y. M., Chen, B., Jiang, J. D., and Zhang, J. P. (2018). Syntaxin 1B mediates berberine's roles in epilepsy-like behavior in a pentylenetetrazole-induced seizure zebrafish model. Front. Mol. Neurosci. 11 (378), 378. doi: 10.3389/ fnmol.2018.00378

Zhu, X., Bian, H., and Gao, X. (2016). The potential mechanisms of berberine in the treatment of nonalcoholic fatty liver disease. Molecules 21, 10. doi: 10.3390/ molecules 21101336

Conflict of Interest: The authors declare that the research was conducted in the absence of any commercial or financial relationships that could be construed as a potential conflict of interest.

Copyright $\odot 2020$ Chen, Zheng, Zhang, Han, Zhang and Hu. This is an openaccess article distributed under the terms of the Creative Commons Attribution License (CC BY). The use, distribution or reproduction in other forums is permitted, provided the original author(s) and the copyright owner(s) are credited and that the original publication in this journal is cited, in accordance with accepted academic practice. No use, distribution or reproduction is permitted which does not comply with these terms. 